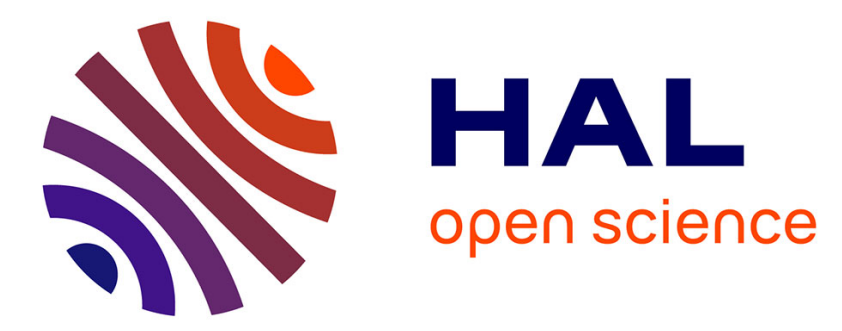

\title{
Current sheets in planetary magnetospheres
}

Lev Zelenyi, Helmi Malova, Elena Grigorenko, Victor Yu Popov, Dominique

\author{
C. Delcourt
}

\section{To cite this version:}

Lev Zelenyi, Helmi Malova, Elena Grigorenko, Victor Yu Popov, Dominique C. Delcourt. Current sheets in planetary magnetospheres. Plasma Physics and Controlled Fusion, 2019, 61 (5), pp.054002. 10.1088/1361-6587/aafbbf . hal-02366108

\section{HAL Id: hal-02366108 \\ https://hal.science/hal-02366108}

Submitted on 20 Nov 2019

HAL is a multi-disciplinary open access archive for the deposit and dissemination of scientific research documents, whether they are published or not. The documents may come from teaching and research institutions in France or abroad, or from public or private research centers.
L'archive ouverte pluridisciplinaire HAL, est destinée au dépôt et à la diffusion de documents scientifiques de niveau recherche, publiés ou non, émanant des établissements d'enseignement et de recherche français ou étrangers, des laboratoires publics ou privés. 


\title{
Current sheets in planetary magnetospheres
}

\author{
Lev Zelenyi ${ }^{1}$, Helmi Malova ${ }^{2,1}$, Elena Grigorenko ${ }^{1}$, Victor Popov ${ }^{3,1,2}$ and Dominique \\ Delcourt $^{4}$ \\ ${ }^{1}$ Space Research Institute of the Russian Academy of Sciences, Moscow, Russia \\ ${ }^{2}$ Scobeltsyn Institute of Nuclear Physics, Lomonosov Moscow State University, Moscow, Russia \\ ${ }^{3}$ Physics Faculty of Lomonosov Moscow State University, Moscow, Russia \\ ${ }^{4}$ CNRS- Universite d'Orleans - CNES, Orlean, France \\ E-mail: lzelenyi@iki.rssi.ru
}

Received $\mathrm{xxxxxx}$

Accepted for publication $\mathrm{xxxxxx}$

Published $\mathrm{xxxxxx}$

\begin{abstract}
In this article, we would like to highlight the problems related to the structure and stability of comparatively thin current sheets, that were relatively recently discovered by space missions in the magnetospheres of the Earth and planets, as well as in the solar wind. These magnetoplasma structures are universal for collisionless cosmic plasma and can play a key role in the processes of the storage and release of energy in space environment. The development of their self-consistent theory for the Earth's magnetosphere, where they were firstly discovered, has relatively long and dramatic history. The solution of the problem of thin current sheet structure and stability become possible in a frame of a kinetic quasiadiabatic approach required to explain their embedding and property of metastability. It was found that the structure and stability of current structures are completely determined by the nonlinear dynamics of plasma particles. Theoretical models developed to predict many properties of these structures and interpret many experimental observations in planetary magnetospheres and heliosphere.
\end{abstract}

Keywords: space plasma, current sheet, energy storage and release

1. Peculiarities of planetary magnetospheres as a result of solar wind - magnetic field interactions

After satellite measurements of $60^{\text {th }}$ and $70^{\text {th }}$ it became evident that the Earth's dipole magnetic field actively interacts with the solar wind (SW) [1]-[4] producing the streamlining flow of the solar plasma with the frozen-in interplanetary magnetic field (IMF). This interaction leads to the formation of the giant magnetic cavity (magnetosphere), surrounding a planet, where SW plasma is deflected by the intrinsic (or induced, for some planets) magnetic field. N. Ness in [5] on the basis of then available experimental observations described the Earth's magnetosphere as nonspherical object like very compressed dipole at the dayside, and non-dipole shaped magnetic structure at the Earth's nightside resembling the cometary tail (see Fig.1). This structure strongly stretched by the solar wind at the night side was named the magnetotail. The Earth's magnetotail seemed to be very elongated structure about $250-300 R_{E}\left(R_{E}\right.$ $\approx 6400 \mathrm{~km}$ is the Earth's radius). In its symmetry plane the electric currents with value about $10^{6} \mathrm{~A}$ are flowing in the direction from dawn to dusk. This current sheet (CS) selfconsistently supports the oppositely directed magnetic fields in a vast region named "magnetotail lobes". The magnetotail has a tendency to flare downstream from the Earth forming approximately the surface of the rotational paraboloid [6]. Observations showed that magnetotail current sheet thickness depends on the state of the magnetosphere [7-9]: in the growth phase of substorms (global magnetic perturbations) magnetotail CS is thinned from about several $R_{E}$ to the thickness about one to several proton gyroradii, i.e. about from 250 to $4000 \mathrm{~km}$ [7]-[11]. It is supposed that this extremely thin current sheet (TCS) in the Earth's magnetotail can play a key role in the development of a substorm cycle 
of magnetic energy "storage-release". CS processes can trigger the switching of the evolutionary dynamics of energy storage in the magnetotail to the explosive-like release of the stored energy due to instability, which can even completely disrupt CS itself[12, 11]. Moreover, the natural turbulence in TCSs is also responsible for the energization of the magnetospheric plasma, particularly, for plasma heating, acceleration, and transport in the magnetosphere.

Due to a numerous modern spacecraft missions it is clear now that TCSs can be observed everywhere in a space plasma. They seem to be a universal structures in a space plasma responsible for energy storage and release, magnetic reconnection, plasma acceleration and other important processes in all planetary magnetospheres, in SW [11, 1316], solar corona [17] and in many laboratory experiments [18].

Planetary magnetospheres are really the result of the supersonic SW flows interaction with intrinsic magnetic fields of planets or their conducting ionospheres. In this sense one can say about the universality of both magnetospheric-type interactions and the scaling factor characterizing their spatial and temporal characteristics in comparison with the Earth's one. One can distinguish the general basic properties of planetary magnetospheres. Firstly, they might have intrinsic source of magnetic field due to internal dynamo processes (Mercury, Earth, giant and ice planets). Secondly they can have induced magnetospheres (Venus and Mars) and in the same time the entirely lost or weak residual magnetic fields. Relatively small planets with a weaker magnetic field like a Mercury might have much more simple structure of magnetosphere in comparison with larger and complicated magnetospheres of the Earth and giant planets. Thirdly, for the majority of magnetospheric magnetotail CSs have usually a flat configuration in the equatorial plane [19]. Axis-symmetric cylindrical current configuration is characteristic for planets which have the pole-on position of their magnetic dipoles relatively to the direction of solar wind flow (e.g. Uranus, Neptune, small planet Pluto) [20-22].

For fast rotating giant planets with strong intrinsic magnetic field the processes of corotation (generation of electric field due to planetary rotation which involves the plasma surrounding the planet to the (co-)rotation with the same angular velocity) and the presence of small satellites as strong source of plasma besides the SW could play a significant role in the formation of specific magnetodisk configurations with strong ring electric current about (90$160) * 10^{6}$ A $[23,24]$. The fine structure of magnetodisk current in the equatorial plane of the planet can not be described fully in a frame of MHD approach [25, 26] because of the small transversal disk thickness. This magnetodisk current can be also a kind of TCS of cylindrical shape that could be considered as a tangential discontinuity in a frame of MHD consideration.

Below we elucidate some aspects of the structure and stability of planetary magnetospheres related to the key universal structures within them, i.e. TCSs playing the important role in the general energy circulation within them [16]. We will consider planets with plane current systems as much more investigated in comparison with distant planets having or quasi-steady or intermittent cylindrical shapes dependent from the tilt angle of their dipole moments relatively to the SW flow.

\section{Magnetospheres of planets: common features and differences}

The comparison of two Earth-type magnetospheric configurations is shown in Fig.1. Hereafter we will use the standard Geocentric Solar Magnetospheric System (GSM), where the $\mathrm{X}$-axis is directed from the Earth center to the center of the Sun, the Z- axis coincides with the direction of the Earth dipole and Y-axis is directed correspondingly from the dawn to dusk side.

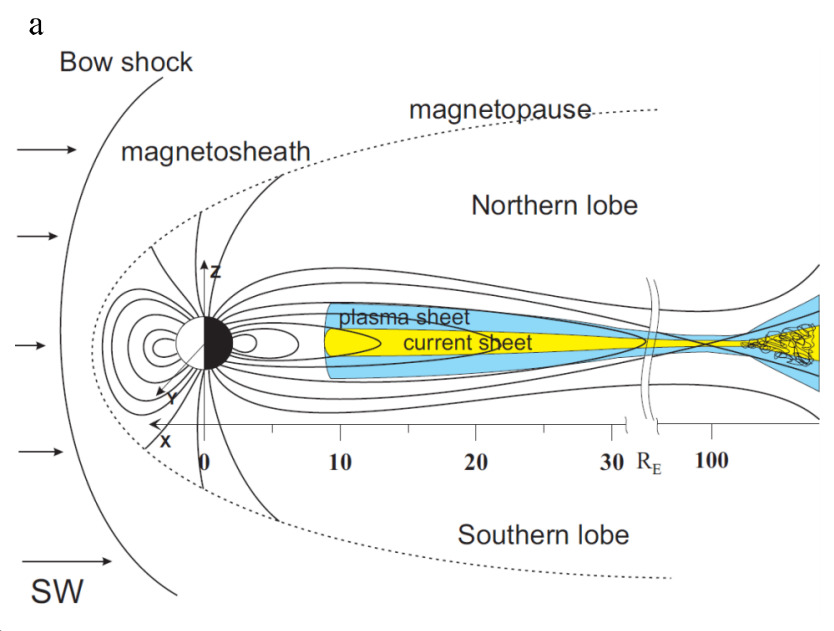

$\mathrm{b}$



Fig.1. Scheme of the Earth's (a) and Mercury (b) magnetospheres (fig $1 \mathrm{~b}$ is adapted from [15]; here black lines are magnetic field lines; colour lines correspond to charged particle trajectories near the planet). 
Streamlining of magnetic dipole by supersonic SW can lead to the formation of a bow shock (standing wave upstream of the magnetosphere), displayed in Fig. 1 for both planets [27]. Magnetopause of the Earth is the very narrow current region with thickness about 100-1600 km (e.g., [28] and references therein) which separates the magnetosphere itself from the SW. In these vast regions TCS with a magnetic field reversed in a neutral plane can be revealed. The transitional region between bow shock and magnetopause is the magnetosheath where plasma motion is strongly turbulent. The shape of the magnetopause is determined by the balance between the dynamical pressure of SW and the static magnetic pressure of the planetary magnetic field [29].

Inner magnetosphere contains radiation belts, representing the regions in the dipole magnetic field with relatively stable trapping of charged particles with energies from about $100 \mathrm{keV}$ to several hundreds $\mathrm{MeV}$. Polar auroral oval is the prominent current system appearing at the boundary between the closed and open magnetic field lines [30]. Fig. 1 demonstrates at the nightside of both planets the stretched magnetic configuration named magnetotail $[5,31]$. Typical plasma density for the Earth is $0.3-0.5$ ions $/ \mathrm{cm}^{3}$ and typical proton energies are of the order of several $\mathrm{keV}$; electron energies are usually 5-8 times lower. Large-scale electric current flows from the dawn to the dusk site and closes over the magnetopause [11]. CS in the neutral plane can stably support this stretched magnetic configuration with a tangential component reversing its sign in the neutral plane [32]. At distance $X \sim-100 \quad R_{E}$ magnetic field lines of the magnetospheric dipole, convecting in the direction of SW flow, can be reconnected at the distant magnetic X-line and move back backward to the Earth; separated IMF magnetic lines move downstream in the tailward direction (Fig.1a). The Earthward convection flow stops off at the distance about 7-9 $R_{E}$ and then plasma flow move around the Earth, forming the close ring current. Sometimes it is considered one more external radiation belt of the Earth, along with abovementioned internal ones. It should be noted that TCSs are usually observed near X-lines in the magnetotail and lie on both their sides. Thus magnetotail can play the role of the energy reservoir where an excess of free magnetic energy gained during substorms might be accumulated in TCS formed in a neutral plane, and then be quickly released in a form of kinetic energy of plasma flows near newly formed reconnection regions. This theoretical concept is supported now rather convincingly by experimental observations [11].

Planetary magnetospheres might be quite different in their dimensions but all of them have the similar topology. Therefore one can suppose that self-similar scaling laws could be applied for their comparison. Taking into account that the density of SW decreases as inverse square of the distance $r$ from the Sun, one can estimate the relative stand off SW distance [33]:

$$
\frac{D_{p}}{D_{E}}=\left(\frac{r_{p} M_{p}}{M_{E}}\right)^{1 / 3}
$$

Here, $D_{p}, D_{E}, M_{p}, M_{E}$ are correspondingly the subsolar radii and dipole moments of planetary magnetospheres (p) and the Earth (E), $r_{p}$ is the heliocentric distance of the planet in astronomical units $\mathrm{AU}$ ( $\mathrm{AU}=$ distance from the Earth to the Sun). This scaling factor (1) has a universal character in the hierarchy of magnetospheric scales and might be applied also to estimate other spatial and temporal characteristics of magnetospheres of different planets and to compare results with available observations.

Now let us consider the peculiarities of the Hermean magnetosphere (Fig.1b), the second planet of the Earth's type. It was a great surprise for scientists that this small iron planet with radius $2400 \mathrm{~km}$ posseses an intrinsic magnetic field [24]. Mariner 10 flybys in 1974-1975 near Mercury demonstrated that the size of its magnetosphere is about $5 \%$ of the Earth's one. The spatial scales of Mercury's magnetosphere, accordingly to scaling law (1) might be estimated relatively to the Earth's spatial distances by a factor of about 8 [34]. Thus the weak magnetic field of the Mercury stops SW flow at the distance about $0.6 R_{M}$ from the surface of the planet. Thus Mercury occupies the most part of its magnetosphere; as a result it has no ionosphere, no radiation belts and no ring current [35]. The Mercury's magnetotail is observed at distance till $10 R_{M}$ at the nightside. Mariner 10 observations have shown that relatively thick lobes of Mercury's magnetosphere are separated by a very thin current sheet with thickness about $150-300 \mathrm{~km}$ [36], that is comparable with gyroradii of protons. Particle motion, as well as their transfer and acceleration in the magnetotail are strongly non-adiabatic and can be described in a frame of quasi-adiabatic approach ([11, 16] and references therein). Generally, due to its proximity to the Sun and smallness, Hermean magnetosphere is strongly driven by SW. Substorms here can occur almost every minute [37].

Contrary to the Earth and Mercury, that have intrinsic magnetic fields, the planetary field of Venus and Mars, schematically shown in Fig.2, are not able to deflect substantially the SW. The nature of the interaction between solar wind and non-magnetized objects depends on the electrical conductivity of the body. The incident plasma stream could be slowed and then deflected by the induced magnetic field if current loop going over the conducting planetary surface and/or its conducting ionosphere to the solar wind is formed in the planetary plasma environment [35]. As a result the magnetosphere-like cavity, named "induced magnetosphere" with elongated magnetotail could be formed around the planet (Fig.2). Thus Mars and Venus do not have large scale intrinsic magnetic fields but have ionospheres providing the conducting paths to close the induced currents and generate such exotic "magnetosphere". There is no distinct boundary like the Earth's magnetopause. 
Ionopause plays the role of a "barrier" separating the planetary plasma from the plasma of SW. The system of shielding induced currents is modified by a comet-like pickup process engaging exospheric and ionospheric ions [38]. General processes there are: 1) photoionization of planetary atoms; 2) mass loading of SW flow by planetary ions; 3) differential draping of magnetic field lines; and 4) formation of magnetotail from field lines bended due to the differential mass loading. These processes play a key role in formation of the induced magnetosphere by IMF field lines convecting around the planet.

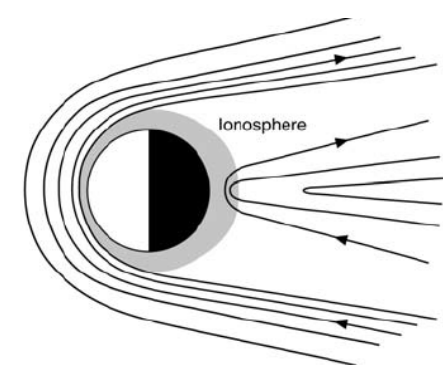

Fig.2. The concept of the induced magnetospheres adapted from [39]. The planet is surrounded by ionosphere supporting the induced magnetic field with elongated magnetotail-like structure in the downstream direction. The ionosphere separates the planetary plasma from the SW. Supersonic flow of solar wind produces the bow shock upstream of the planetary ionosphere representing the conducting obstacle.

Global magnetic perturbations are characteristic for the induced magnetospheres of Mars and Venus, depending on their interaction with the SW plasma flow. It was shown that in analogy with the Earth's magnetic storms a significant magnetic activity also takes place at Mars [40]. Martian magnetic storms are found to be associated with passage of the planet across the heliospheric current sheet, accompanied by changes of IMF polarity. Mars Express measurements have shown that during Martian magnetic storms strong perturbations in magnetosphere and ionosphere occur. The magnetic barrier formed by the pile-up of IMF in front of the ionopause decays and does not provide any shielding from the incoming solar wind. Large blobs (clouds) of solar wind plasma might penetrate to the Martian magnetosphere and extinct the dense plasma from the ionosphere. The topside region of ionosphere becomes very fragmented and consists of intermittent cold/low energized plasmas [41].

Quantitative model of Venus magnetosphere was developed by Vaisberg and Zelenyi in [42] who followed the dynamics of interplanetary magnetic field lines loaded by heavy planetary picked up ions. While central parts of field lines flowing through the Venus exosphere are mass loaded and decelerated, their ends immersed into SW flow are moving with unperturbed SW velocity. This results in strong bending of interplanetary magnetic field lines and formation of the field reversal region at the nightside resembling the magnetotail. Such tail is formed from open mass-loaded field lines and is named "accretion magnetotail".

The magnetotail of the induced magnetosphere of Venus was investigated by Venus Express spacecraft. It was shown that magnetic field of Venus is quite dynamic indicating that reconnection processes in magnetotail can also take place. This could explain the presence of energized ions with energies from 1500 to $2000 \mathrm{eV}$. Nevertheless sometimes magnetotail magnetic measurements revealed a very quiet tail with the thin current sheet in the central part populated only by low energy ions [43].

Due to successful missions of Pioneers 10, 11, Voyagers 1, 2 and Galileo spacecraft the basic properties of Jovian and Saturnian magnetospheres are known in general. Jupiter is the largest planet of Solar system (radius $\mathrm{R}_{\mathrm{J}} \approx 71400 \mathrm{~km}$ ); it has the strongest magnetic field with the magnetic moment 18000 times larger in comparison with the Earth's one. The Jovian magnetotail extends in the antisunward direction at 650 million $\mathrm{km}$ reaching the orbit of Saturn [35]. The structure of this magnetosphere is more complex than the Earth's one, containing bow shock, magnetopause, magnetotail and magnetodisk (Fig.4). Jovian bow shock is located at the average distance about $\sim 7 * 10^{6}$ $\mathrm{km}$ from the center of planet; the magnetopause is observed at a distance about $60-70 \mathrm{R}_{\mathrm{J}}$.

Because of the additional amount of plasma from satellite Io, known by its volcanic activity, the size of Jovian magnetosphere is substantially expanded and gives an additional component to the pressure balance against the SW pressure. At the night side of the planet, the magnetosphere of Jupiter is stretched into a long magnetotail similar to the Earth's one; two lobes are separated by a thin current sheet in the center. The magnetotail current sheet is closed on the magnetopause due to Chapman-Ferraro currents.

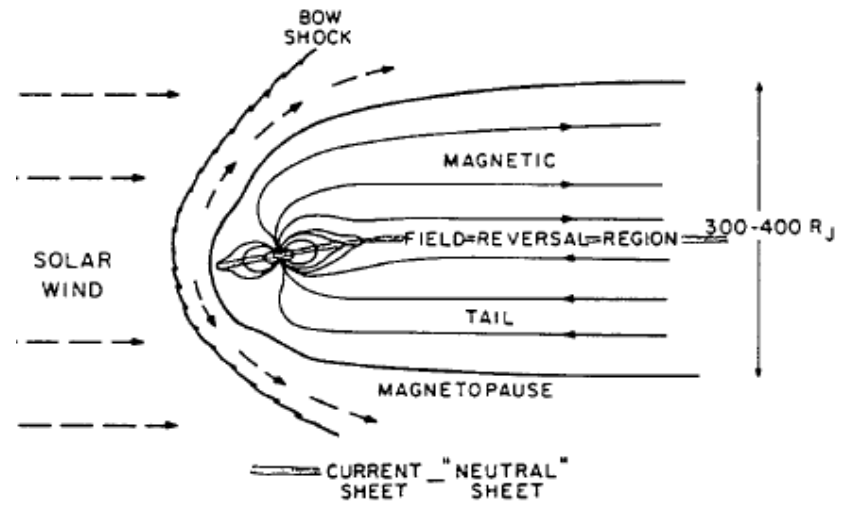

Fig.3. Schematic view of the structure of the Jovian magnetosphere [44].

It was proposed in [45] that steady state reconnection at the external edges of the Jovian magnetodisk 
should support the formation of plasmoids and return of empty flux tubes to the inner magnetosphere.

Galileo observations indicated that beyond $40 \mathrm{R}_{\mathrm{J}}$ the Jovian magnetodisk current sheet is disrupted and beyond 50 $\mathrm{R}_{\mathrm{J}}$ at the nightside tail magnetic field could be explosively reconnected [44]. These disruption events are not regular; they have the characteristic time scales from 4 hours (for small events) to about $24 \mathrm{~h}$ (for large events). Because of these reconnection processes plasmoids enter into the Jovian tail and provoke fast reconnection in magnetotail current sheet at the nightside of the planet. This could support the close plasma and magnetic flux circulation around the planet. These plasma disturbances could be interpreted as Jovian substorms. Therefore, unlike the Earth's like planets the Jovian substorms depend substantially on the internal convection and are only slightly driven by SWmagnetosphere interaction.

Summing up this section, one can say that the magnetosphere of the planets which have or do not have their intrinsic magnetic field, can possess not only differences but also many similarities. Magnitude of the planetary magnetic dipole field interacting with the supersonic SW flow determines the scaling factor (see eq. (1)) describing the hierarchy of magnetospheric scales relatively the Earth's one. In turn, the sizes of the magnetospheres determine their relationship to the $\mathrm{SW}$ driving. The larger the magnetosphere (e.g., Jupiter), the more magnetospheric structures and processes are less driven by the SW. The smaller the magnetosphere (Mercury, Earth), the more it depends on transient SW flows. Magnetospheres are shielded from the SW by a complcated system of currents flowing along their boundary structures (bow shocks, magnetopauses, magnetotails). Thin current sheets seem to be universal structures characteristic for all magnetospheres where the storage and release of energy takes place as a result of global perturbations, storms and substorms. Below we consider in more details the properties of current sheets in planetary magnetotails and their consequences for the planetary environments and energy balance.

\section{Experimental observations of multiscale current sheets as universal structures in space plasma.}

Spacecraft data in the Earth's magnetosphere obtained onboard of Geotail, Interball, Cluster and other spacecraft provided an unprecedented opportunity to receive a new knowledge and develop a new generation of models to investigate current sheets in the magnetosphere. In recent years the attention of many researchers has been focused on extremely thin current sheets with thicknesses about ion Larmor radius or less, which were observed in the magnetotail at different distances from the Earth [7-10]. The region at the near-Earth edge of the magnetotail current sheet is of particular interest, because, first of all, it is more easily accessible by spacecraft, secondly, it is the presumed domain of substorm initiation, explosive-like evolution and energy transformations. TCSs in this region may appear as a result of the enhanced plasma convection in the course of the substorm growth phase and, as we have mentioned above, might play a role of the reservoir of magnetic energy, which could be released after current sheet disruption at the onset of substorm expansion phase.

The structure and properties of thin current sheets in the Earth's magnetosphere are investigated now by many spacecraft-borne "in situ" experiments, including recent multi-point Cluster measurements [10]. The essential properties of TCS are different from those of current sheets which are observed under the quiet conditions in the magnetosphere. Satellite investigations revealed the following particular properties of TCS:


Fig.4. Two examples of characteristic profiles of magnetic field and current density in the Martian magnetotail from Maven observations [46] .

1) Very small thicknesses $L \sim \rho_{p} \sim 250-1000 \mathrm{~km}$, where $\rho_{p}$ is the proton gyroradius. Such thin current profiles are often observed in the Earth's magnetosphere [7-12, 29, 43]. Fig.4 demonstrates two characteristic crossings of the Martian magnetotail [46]. Here one can clearly see, especially in Fig.4b that current density profiles have a multiscale embedded character: extremely thin current sheet (with the scale less than $100 \mathrm{~km}$ ) is embedded inside thicker current sheet with the thickness about $400 \mathrm{~km}$. Fig. 5 shows current sheet intersection by spacecraft Venus Express in the Venus magnetotail [47] (a) and by Mariner 10 in the Mercury one [48]. At the last case the time scale of CS crossing was $40 \mathrm{~s}$, jump of the magnetic field was $80 \mathrm{nT}$. The thickness of current sheet observed, probably, after dipolarization was estimated as $150 \mathrm{~km}$. For comparison, the gyroradius of proton with energy $2 \mathrm{keV}$ is about $1000 \mathrm{~km}$ in the magnetic field with the magnitude $40 \mathrm{nT}$. 
2) Large value of current density, which might be 10-20 times larger than the one under usual tail conditions $(\sim 10$ $\mathrm{nA} / \mathrm{M}^{2}$ for the Earth).
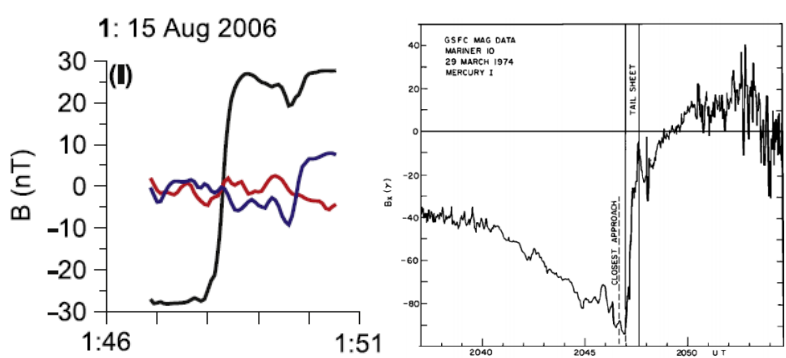

Fig. 5. Current sheet crossings (a) by Venus Express in the Venus magnetotail [47] and (b) Mariner 10 data in Mercury magnetotail current sheet [48].

3) Very stretched shape of magnetic field lines, with $B_{z} / B_{x} \sim 0.1$.

4) Ions at the open (so called Speiser orbits ) are usually the main current carriers across the sheet $[11,16]$. Electron currents can dominate in the narrow region in the neutral plane and are produced presumably by drift currents [49].

a
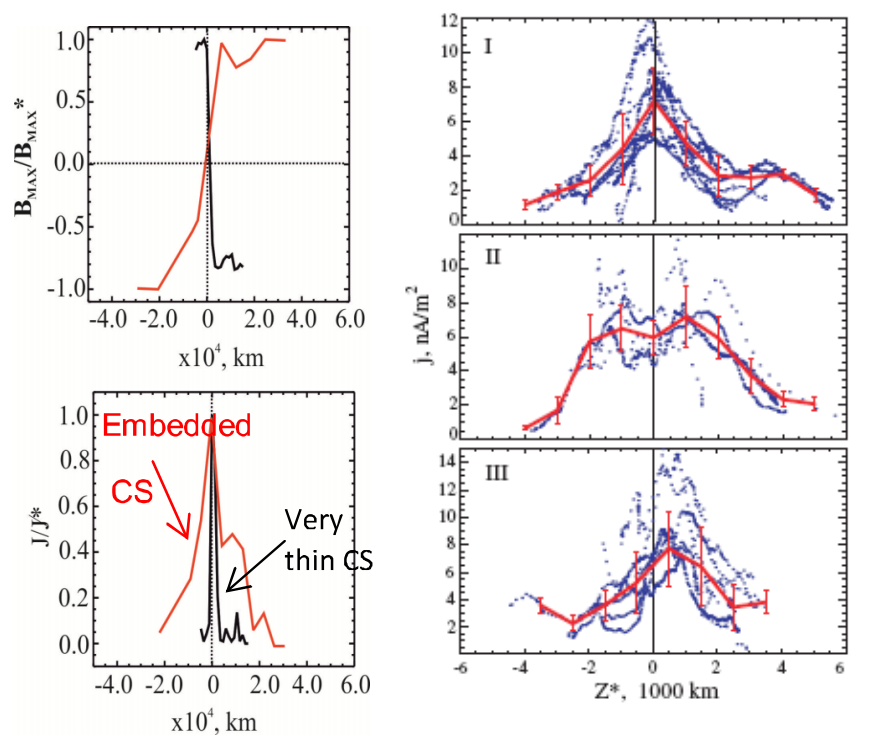

Fig. 6. Different observations of TCS in the space plasma: (a) embedded strong current sheets in the solar wind observed within the heliospheric plasma sheet (adapted from [50]); (b) average vertical profiles in the Earth's magnetotail for center peaked (I), bifurcated (II) and asymmetrical current sheets (III) [51].

5) Current density profile usually does not coincide with plasma density one: TCS is embedded within more thick plasma sheet $[11,16]$. It is shown on the basis of ISEE 1 and ISEE2 observations in the Earth's magnetosphere that TCSs with thicknesses about $0.2 R_{E}$ were found to be embedded into much broader plasma sheet with the transverse scale $\mathrm{L} \sim 3-4 R_{E}$. Multiscale character of multiple TCSs in the region of heliospheric plasma sheet in SW was suggested by Stereo-A observations [50], shown in Fig.6a.

6) Cluster data [51] showed that TCS can have not only classical bell-shape current profiles, but these profiles could be bifurcated, with two maxima of current density at the edges and minima in the center (see Fig. 6b from paper [51]. 7) In some observations one can see unusual sheets with current peaks shifted from zero of the magnetic field and having asymmetrical shape of current density (fig.6, III). The reasons of this asymmetry were studied, e.g. in [52, 53].

8) TCSs are metastable, i.e. after its formation they remain stable during the entire growth phase of substorms(time periods in the Earth's magnetotail from 15 min to 2 hours) and after it they can be explosively destructed in the course substorm expansion phase resulting in particle thermalization and acceleration as well as in significant enhancement of wave activity $[10,11,13,46,54]$.

\section{TCS as reservoirs of magnetic energy}

The idea that solar flares are the result of energy release due to disruption of current sheets was developed by Syrovatsky in [17]. Accumulated magnetic energy can be transformed in solar flares both to the thermal and bulk energy of particle motion. Now it is known that magnetic reconnection in thin current sheets formed due to crossing of magnetic loops is responsible in solar corona for solar flares. Generally TCSs in the heliospheric and magnetospheric plasma are the places of the transformation of stored magnetic energy into kinetic energy of plasma particles, their transport and acceleration. Because TCS separates two regions with oppositely directed magnetic field lines, their reconnection usually leads to current sheet disruption and corresponding sheet filamentation [54].

The collisionless tearing (current filamentation) instability is the most probable mechanism initiating the beginning of magnetic reconnection in TCS, consequent plasma acceleration and plasmoid formation. Theoretical analysis in [55] showed that Harris-like current sheet [56] with the only one tangential magnetic component $B_{x}$ is always unstable for tearing mode. But later it was shown [57] that the effect of the electron compressibility prevents the development of a tearing mode in a model of Harris-like current sheet with a small normal magnetic field component (this small $B_{z}$ component is always unavoidably persistent in planetary magnetotails). Sufficiently strong electrostatic field appears to support charge neutrality of the perturbed system because electrons (contrary to ions) are magnetized by $B_{z}$ field. Thus the energy of perturbed electrons exceeds the available free energy of current sheet, preventing the development of tearing mode [58]. 
The problem of a full stability of collisionless tearing mode in $\mathrm{CS}$ with nonzero normal magnetic component became a theoretical mystery for several decades. Many efforts were made to make models of magnetotail configuration unstable taking into account e.g. kink and other modes (see e.g. [11, 13, 59-61] and references therein). It have been shown in these studies that all these instabilities either develop very slowly or saturate at a very low level. All these early studies of tearing mode were based on CS models with nonzero normal component assuming at the same time isotropic distribution functions of plasma components. All attempts to expand the instability region considering the mechanisms of plasma microturbulence, chaotic scattering, bending, coupling with other modes like ballooning $[11,13]$ were not successful. Multipoint observations of the spacecraft missions in the tail of Earth's magnetosphere, later in other planetary magnetospheres and in the SW resumed the interest to the stability of CSs, since these observations confirmed that current sheets discussed in early papers by Syrovatskii [17] can be observed not only in planetary magnetospheres, but also in the SW and solar corona. In the paper [63] the solution of this paradox of a full stability of Harris CS with $\mathrm{Bz}$ was outlined and the tearing stability of a more suitable and realistic quasi-adiabatic model was investigated. Particularly, the most important property of metastability was explained in a frame of this model. Later other kinds of unstable modes (king, sausage, oblique tearing, drift et al.) were investigated [64]. Below we present TCS quasi-adiabatic model which can explain the observational features of TCS structure and dynamics.

\section{Quasi-adiabatic motion and equilibrium CS} models

In the simplest 1D collisionless current sheet model (Harris, 1959) the shifted Maxwellian distributions for both ions and electrons were considered:

$$
\begin{aligned}
& f_{\alpha}(v)=n_{\alpha}(z)\left(\frac{m_{\alpha}}{2 \pi T_{\alpha}}\right)^{3 / 2} \exp \left\{-m_{\alpha} \frac{\left(v_{x}^{2}+\left(v_{y}-V_{\alpha y}\right)^{2}+v_{z}^{2}\right)}{2 T_{\alpha}}\right\}, \\
& n_{\alpha}(z)=n_{0} \exp \left(e_{\alpha} V_{\alpha y} A_{y}(z) / c T_{\alpha}\right), \\
& \frac{d B_{x}}{d z}=\frac{4 \pi}{c} \sum_{\alpha=i, e} \int e \vec{v} f_{\alpha}(\vec{v}, z) d \vec{v}, \sum_{\alpha=i, e} e_{\alpha} n_{\alpha}=0, \varphi(z)=0,
\end{aligned}
$$

where $T_{\alpha}$ is the temperature of the $\alpha=i, e$ kind of particles, $m_{\alpha}$ is the mass, $V_{\alpha y}$ is the flow velocity, $W_{0}=m_{\alpha} v^{2} / 2$ is the total particle energy, $P_{y}=m_{\alpha} v_{y}+(e / c) A_{y}(z)$ is the generalized momentum. Corresponding self-consistent profiles of current, magnetic field and plasma density have the form:

$B_{x}(z)=B_{0} \tanh (z / L), n(z)=n_{0} / \cosh ^{2}(z / L), j_{y}(z)=J_{0} / \cosh ^{2}(z / L)$ where half-thickness of current sheet is equal to $L=2\left(T_{i}+T_{e}\right) /\left\{c B_{0}\left(V_{i y}-V_{e y}\right)\right\}$. Characteristic property of such $\mathrm{CS}$ is that the profiles of current and plasma density coincide, because their transverse scale is the same.
First 2D model of magnetotail current sheet was proposed in [65] for the description of relatively thick magnetotail CS with fully magnetized plasma particles. The general approach that was applied is the MHD approximation where the corresponding tensor of plasma pressure is assumed to be isotropic. In such models the tension of the magnetic field lines is balanced by the gradient of plasma pressure in the Earth-Sun direction. The semifluid approach supposes CS anisotropy with diagonal pressure tensor [66]. All these models can not be applied within TCSs where guiding center approximation is violated.

The pioneering analysis of particle dynamics in the neutral plane with strong transverse magnetic gradients was published by Speiser in [65]. It was shown that in such CSs protons are demagnetized in the neutral plane, but their quasi-adiabatic integral of transverse (along $z$ coordinate) oscillations $I_{z}=(1 / 2 \pi) \oint p_{z} d z$ is approximately conserved.

This kind of particle motion was later named as quasiadiabatic [67]. Contrary to protons in TCSs electrons are fully magnetized, therefore the dynamics of different plasma particle species is drastically different. The models of extremely thin current sheet with Speiser's ions as the main current carriers were developed later in [68-70]. The work [70] demonstrated that electrons can be current carriers together with quasi-adiabatic protons, due to a strong gradient drift currents, but they sensitively depend on the curvature of magnetic field lines related to the value of the normal magnetic component. The new type of extremely thin plasma equilibria with thickness $L \sim \rho_{L}$ (where $\rho_{L}$ is the ion gyroradius) could not be supported by longitudinal pressure gradients but only by the off-diagonal terms of the pressure tensor. It was shown that these $1 \mathrm{D}$ magnetic configurations are almost homogeneous along Earth-Sun direction [71]. Contrary to isotropic models of relatively thick current sheets (both MHD and kinetic one), plasma pressure in TCS models is principally non-gyrotropic. It was shown in numerical models of TCS [72] and later supported by experimental observations [73] that velocity distribution functions of ions in TCSs have non-isotropic, non-gyrotropic and nonMaxwellian pressure tensor. The characteristics of anisotropic pressure tensor in self-consistent TCS model were investigated in details [74] and were in good agreement with an earlier results [72]. Generally because of TCS small thicknesses and corresponding violation of ion guiding center motion their description goes beyond the MHD validity limits. In this paper we will describe mostly models using kinetic description of particle dynamics as a most adequate to TCS description.

Particle dynamics in the reversed magnetic field of TCS is determined by the parameter adiabaticity $\kappa$, characterizing the ratio of minimum curvature radius of magnetic field line $R_{c}$ to the maximum Larmor radius $\rho_{L}$ 
near the neutral line: $\kappa=\sqrt{R_{c} / \rho_{L}}$. Parameter $\kappa$ is the key one in particle dynamics. At $\kappa \gg 1$ plasma particles are fully magnetized and their motion can be described in a guiding center approximation. At $\kappa \sim 1$ particle motion becomes essentially nonlinear [75]. At $\kappa \ll 1$ particle gyroradii become about curvature radii of magnetic lines and in this transitional regime particle motion becomes chaotic. For $\kappa<1$ particle motion becomes quasi-adiabatic: usual invariant-magnetic moments is not conserved at this regime but the invariant of motion $I_{z}=(2 \pi)^{-1} \oint p_{z} d z$ could be considered as being approximately conserved [67]. In these regime particles are still magnetized outside CS but are demagnetized in the neutral plane and are moving along serpentine-like orbits, alternately crossing the CS moving from above and below. Within TCS X and Z degrees of freedom of serpentine motion are decoupled: particles perform fast vertical oscillations in $\mathrm{Z}$ direction and simultaneous slow rotation in the $X Y$ plane. Near the separatrix separating two types of motion the small jump of integral of motion $\Delta I_{z}$ takes place so that $\Delta I_{z} \ll I_{z}$. It was shown that DELTA Iz is proportional to the value of parameter $\kappa$ [16]. Together with Speiser particles having small values of invariant of motion $\mathrm{Iz}$ and moving along so called transient trajectories, there exist so called quasitrapped population of particles that, contrary to Speiser's ones, go along quasi- closed orbits and can cross CS many times, their $I_{z}$ invariants are larger than for Speiser's one [11]. Because the average square value of the jump $\Delta I_{z}$ is not equal to zero CS crossing by quasi-trapped particles can be described as a diffusive process with a coefficient of diffusion: $D=\left\langle\left(\Delta I_{z}\right)^{2}\right\rangle / T_{Q T}$, where $T_{Q T}$ is the approximate time scale of the cycle of quasi-trapped particle motion. This process accompanied by the accumulation of quasi-trapped plasma was called "the CS aging" [76]. In the magnetotail electron parameter $\kappa_{e} \sim 2-3$ while for ions this value is one order of magnitude smaller: $\kappa_{i} \sim 0.1-0.2$. This determines the principal difference of dynamics of both particle species and resulting peculiarities of TCS itself. The motion of two plasma components can be described only in a frame of hybrid approach, when protons are described as quasiadiabatic ones and electrons for example as an anisotropic conducting fluid.

Analytical self-consistent model of TCS, taking into account both quasi-adiabatic protons and magnetized electrons was proposed firstly in [70]. TCS is so thin in comparison with scales in $\mathrm{X}$ and $\mathrm{Y}$ directions that one can consider it as a $1 \mathrm{D}$ configuration, with all characteristics depending only from $\mathrm{Z}$ coordinate. There was considered simplified magnetic field $\boldsymbol{B}=\left\{B_{x}(z), 0, B_{n}\right\}$, where tangential component $B_{x}(z)$ reverses sign across the equatorial $z=0$ plane (see fig.1). The normal component $B_{n}$ is supposed to be constant. Thus particle flows comes from the edges of the system (which corresponds in reality to plasma mantle source) towards the neutral sheet plane $(z=0)$. Ion population is supposed to consist of transient and quasitrapped particles with distribution functions, correspondingly, $f_{\text {trans }}(\vec{v}) \sim \exp \left\{-\left[\left(v_{\|}-v_{D}\right)^{2}+v_{\perp}^{2}\right] / v_{T}^{2}\right\} d \vec{v}$ and $f_{\text {trap }}(\vec{v}) \sim \exp \left\{-v_{0}^{2} / v_{T}^{2}\right\} d \vec{v} \quad\left(v_{T}\right.$ and $v_{D}$ designate the thermal and bulk flow velocities, $v_{0}$ is the total speed). Ion population is described by the quasi-adiabatic approach so that the action integral of ion motion $I_{z}=(m / 2 \pi) \oint v_{z} d z$ could be considered as approximately conserved. Electron dynamics is supposed to be fast enough to support a quasi-equilibrium Boltzmann distribution in the presence of an ambipolar electrostatic potential and mirror forces. Plasma quasineutrality $n_{i} \approx n_{e}$ is supported by these electrostatic fields. Such approach generalizes earlier quasi-adiabatic model [77, 78] where electrostatic effects were not taken into account. The model of TCS is described by the self-consistent system of Vlasov-Maxwell equations:

$$
\frac{d B_{x}}{d z}=(4 \pi / c)\left(j_{y i}(z)+j_{y e}(z)\right)
$$

where ion current is the sum of currents carried by transient and trapped particles: $j_{y i}=(4 \pi / c) \sum_{i} e \int v_{y} f_{i}(z, \vec{v}) d \vec{v}$. Here $f_{i}$ is the local $i$-th ion distribution function that can be extrapolated to the entire CS domain by its mapping from the edges of TCS to the neutral plane using quasi-adiabatic integral of motion $I_{z}$ and applying the Liouville's theorem. Finally, the generalized distribution functions of transient Speiser's and quasi-trapped particles acquires corresponding forms: $f_{\text {trans }}(\vec{v}) \sim \exp \left\{-\left(\sqrt{v_{0}^{2}-\left(\omega_{0} / m\right) I_{z}}-v_{D}\right)^{2}+\left(\omega_{0} / m\right) I_{z} / v_{T}^{2}\right\}$ and $f_{\text {trap }}(\vec{v}) \sim \exp \left\{-v_{0}^{2} / v_{T}^{2}\right\}$. Taking into account the

Boltzmann approximation for electrons, their transverse electron drift currents can be calculated in a guiding center approximation:

$j_{e}=-e n_{e} c[\vec{E} \times \vec{h}] / B+\frac{c}{B}\left[\vec{h} \times \vec{\nabla}_{\perp} p_{\perp e}\right]+\frac{c}{B}\left(p_{I l e}-p_{-e}\right)[\vec{h} \times(\vec{h} \vec{\nabla}) \vec{h}]$,

where $\vec{h}=\vec{B} / B$. Expressions for the electron pressure could be found with the help of Chou-Goldberger-Low approximation for perpendicular direction and energy conservation for the parallel one.

Numerical solution of Vlasov-Maxwell system of equations shown above demonstrates multiscale character of TCS, when very narrow electron-dominated CS is embedded inside a significantly y thicker proton-dominated CS. Fig. 7 shows the total current density, as well as separate electron and proton profiles (a). Corresponding profiles of magnetic components are shown in Fig. $7 \mathrm{~b}$. 
If plasma is multicomponent, particularly it contains among with electron and protons a population of a heavy, for example, an oxygen ions (typical for the post-substorm magnetotail), the profiles of current density and magnetic fields acquire the additional scaling related with the additional current sheet with dominating oxygen populations. The whole multiscale current sheet would be embedded inside the thickest plasma sheet [79].

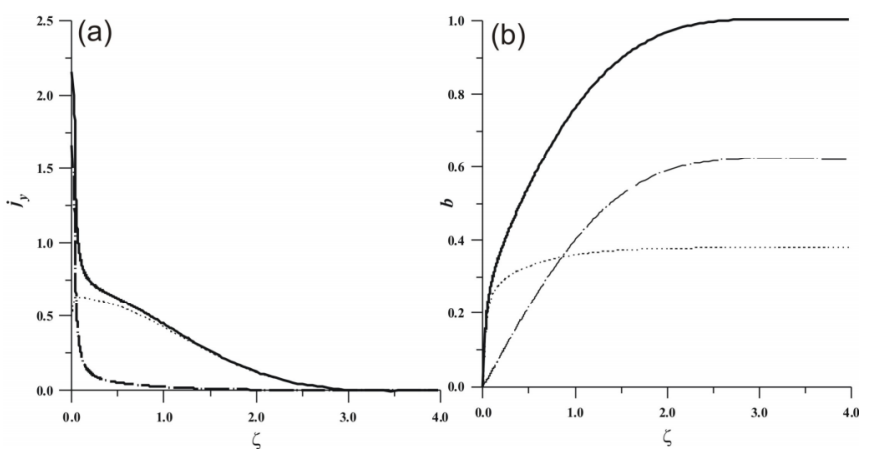

Fig.7. Self-consistent profiles of TCS [49]. At the left one can see the total current density (continious line) and both electron (dotted -dashed line) and ion (dotted line) in dimensionless variables: z-coordinate $\zeta=z \omega_{0} / \varepsilon^{4 / 3} v_{D}$, current density $j_{y}(\zeta)=j_{y} \varepsilon^{-2 / 3} /\left(e n_{0} v_{D}\right)$ and magnetic field $b=B(z) / B_{0}$, where $B_{0}$ is the value of $B_{x}$ at CS edges, $\varepsilon=v_{T} / v_{D}$ is the parameter characterizing ion flow, $v_{T}$ and $v_{D}$ are correspondingly the thermal and flow velocities.

The temporal evolution of TCS contaminated by scattered quasi-trapped plasma (abovementioned TCS "aging" process [76]) is shown in Fig. 8. Due to peculiarities of quasi-trapped particle dynamics these particles support the local negative current in the CS center and positive one at its edges. At the same time their net current is exactly zero because of the closeness of their orbits. The trapped particles can redistribute the current of transient Speiser particles that are main current carriers in TCS. Once the density of quasitrapped particles becomes sufficiently large, current density profile from bell-like shape is transformed to the doublehumped one. The corresponding magnetic field profile demonstrates its flattening with the formation of a smoother profile near the neutral plane. Later these results were confirmed by observational data in the Earth's magnetotail $[10,80]$. Several current sheet crossings by Cluster spacecraft with possible influence of quasi-trapped plasma leading to the formation of smoothed (b) and double-humped (c) current density profiles from single-peaked (a) are shown in Fig.9.

Therefore one could observe the large variety of CSs profiles in a space plasma. Electron drift currents [49], the presence of oxygen heavy ions [79] and the accumulation
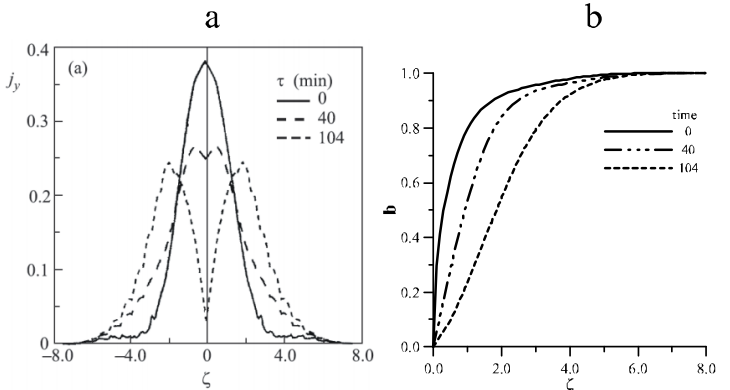

Fig.8. The snapshots of the temporal changing of TCS current density (a) and magnetic field (b) during the "aging" of CS. The values of variables are dimensionless, as in Fig. 8.

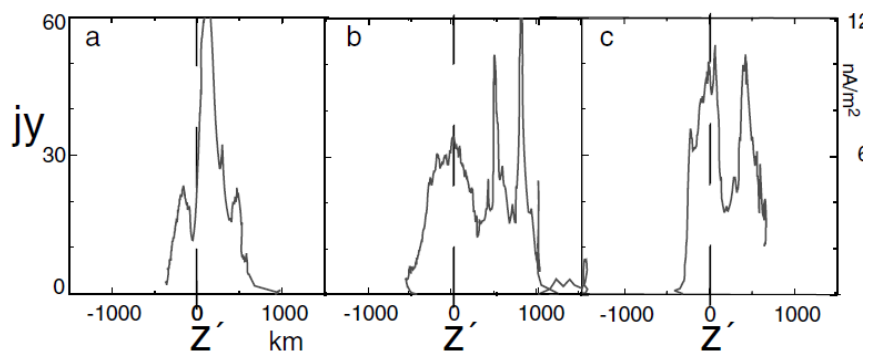

Fig. 9. Reconstructed current sheet profiles of the tail current during TCS crossing (accordingly the work [80]): (a) peaked, (b) flattened and (c) double-humped profiles.

of the quasi-trapped plasma in TCSs [76] represent realistic factors that influence the formation of complex multiscale profiles with several embedded current sheets with different thicknesses in a different planetary magnetospheres $[22,46,47]$. Also such factors as the shear magnetic component or the natural fluctuations of plasma sources can lead to the formation of the asymmetry of TCS current density profiles $[11,53,81]$. All these TCSs configurations can have different structures and conditions of stability because they are mostly determined not by macroscopic characteristics of plasma flows but the peculiar kinetic effects operating within TCS.

\section{Current sheet dynamics}

Tearing ( current filamentation) instability as the key factor of substorm activity of the Earth's magnetotail was proposed many years ago in paper [55] where 1D Harris sheet equilibrium solution have been used for stability analysis. Later Schindler in [82] have shown that even small normal component $\mathrm{Bz}$ in $\mathrm{CS}$ can completely destroy the resonant Landau damping on electrons. Strong stabilizating effect of electrons magnetized by the small but finite normal component of magnetic field resulted in the loss of interest to the tearing as a potential trigger of the onset of the expansion phase of substorms [82]. Neither stochastic motion [67], nor whistler pitch angle scattering were unable 
to overcome the strong stabilizating effect of magnetized electron compressibility [57]. The ideas of possible tearing destabilization due to transient electrons and current driven instabilities were elaborated later [54, 63]. Many other sophisticated possible routes to substorm activization were proposed to solve this problem $[11,13]$. However, the main confusion in practically all earlier papers was related with the fact that all conclusions concerning the absence of tearing mode growth in CSs (see, e.g. [55, 57, 82, 83] were based on the stability analysis of Harris-like current sheet model. Both in experimental observations $[10,51,80]$ and in the CS theory it was found that realistic CSs are principally different from Harris-like configurations. Also due to PIC modeling of collisionless reconnection (see [84]; reviews $[11,13]$ and references therein) it was demonstrated that the strong anisotropy of velocity distribution is characteristic for CSs, contrary to isotropic Harris-like sheet. Therefore one should find the additional factors controlling the stability of CS. Importance of distribution function anisotropy was investigated in papers about CS dynamics [59, 85, 86]. These results make the problem of analytical analysis of stability for anisotropic equilibria very actual. Below we will demonstrate the results of this analysis where principal elements of theoretical consideration in [82] were modified and adaped to a new 1D TCS anisotropic configuration.

The equation for the perturbation of the vector potential $A_{1 y} \sim \exp (\mathrm{i} k x-\mathrm{i} \omega t)$ can be found from the energy principle in which the functional of the perturbed energy of tearing mode

$W_{\text {tearing }}=\int_{-\infty}^{\infty}\left\{\left|\nabla \times A_{1}\right|^{2}+\left|\nabla \varphi_{1}\right|^{2}+\frac{4 \pi}{c} \frac{\partial j_{y}}{\partial A_{0}}\left|A_{1}\right|^{2}+4 \pi e \int_{-\infty}^{\infty} \frac{\widetilde{f}_{1 e}^{2}}{\partial \widetilde{f}_{0 e} / \partial \varphi_{0}} d \vec{v}\right\}$ (4)

should be minimum, i.e.

$$
\delta W_{\text {tearing }}\left(A_{1}\right) / \delta A_{1}=0 .
$$

This allows determining the boundary of a marginal stability of CS considered up to the second order of perturbation theory (as in [82]). Perturbed energy of tearing mode contains three general terms: energies of perturbed magnetic and electric fields (first and second terms), free energy of configuration driving current filamentation $\sim \partial j_{y} / \partial A_{0}$ and the term describing the compressibility of electron fluids $\sim \tilde{f}_{1 e}^{2} / \partial \tilde{f}_{0 e} / \partial \phi_{0}$. Finally condition (5) can be reduced to differential equation for the vector potential of perturbed magnetic field:

$$
\frac{d^{2} A_{1}(z)}{d z^{2}}-\left(k^{2}+\frac{4 \pi}{c} \frac{\partial j_{y}}{\partial A_{0}}+4 \pi \frac{n_{0 e} T_{e} k^{2}}{B_{z}^{2}}\right) A_{1}(z)=0
$$

Functional $\delta W$ (4) was minimized for wave number of perturbation $k$ corresponding to a marginal stability condition $\delta W\left(A_{1 y}\right)<0$. The being the solutions of eq. (6) functions $A_{1 y}(\mathrm{z})$ were found.

This allowed to obtain the parametric map of stable and unstable regions. Tearing mode stability map for governing parameters $\varepsilon$ and $b_{n}$ is shown in Fig.10a. Unstable TCS domains where $\delta W<0$ for at least one value of $k$ were filled by black color. As it is shown at this map the most unstable CSs have strong anisotropic value $\varepsilon<1$ and optimal interval of parameter $b_{n} \sim 0.1-0.2$.

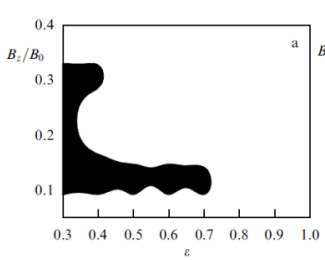

b

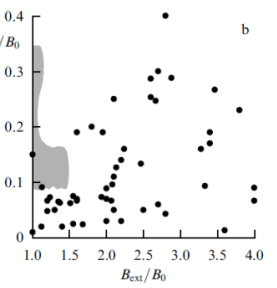

Fig.10. Parametric space of tearing stability of TCSs [13] : (a) A parametric map in the theory. Black color shows the unstable region. (b) Map of the instability regions with the positions of the TCSs observed by Cluster [88]; (c) A parametric instability map with the trajectories corresponding to $\mathrm{CS}$ evolution during the substorm growth phase.

This result has given a chance to revisit an old paradigm associating substorm activity with the onset of tearing-type instability. The idea that magnetotail CSs can become unstable only in some narrow regions in a parameter space was for the first time formulated in [58]. In the work [63] this effect is revisited and investigated in details. in a frame of adequate quasi-adiabatic model of TCS fully suppoerted by modern "in situ" observations.

Therefore one can conclude that the thinning of magnetotail CS is followed by increasing anisotropy of ion and electron distributions and initially isotropic and stable quite thick CS might be transformed into a new metastable equilibrium where CS might experience topological changes enabling the process of fast reconnection. Figure $10 \mathrm{~b}$ demonstrates the parametric space corresponding to Fig.10a [88] where one can see characteristics of CSs observed by Cluster during substorms. Inside the light-black regions the system is unstable and CSs will be destroyed. As a result we see quite dense multitude of TCSs outside this region and small quantity of TCS inside this region. Fig.10c shows the hodograms of TCS states during substorms. Because of a slow CS thinning, its parameters are changed, One can see that all hodograms are streaming from the right upper corner side towards the instability region.

\section{Conclusions}

In this paper a short comparative analysis of planetary magnetospheres was done. It is shown that their self-organized formation in the solar wind is the result of the interaction of the internal planetary magnetic field with the 
incoming supersonic SW flow. As a result of this interaction, the planetary magnetospheres are usually compressed at the day side and strongly elongated like a cometary tails at the night side. What is interesting, even the planets without intrinsic magnetic field, also form a kind of magnetosphere due to the induced currents flowing in the ionosphere and supporting the the current system forming the tail-like structure. Finally depending on the value of planetary magnetic field (including planets without any magnetic field), these magnetospheres from more or less complex structure inside.

Thin current structures are observed in magnetospheres practically everywhere (on the magnetopauses, shock waves, in the tails) and play an important role of reservoirs of free magnetic energy necessary for the beginning of magnetic reconnection. The TCS hybrid model [49] based on the quasiadiabatic proton dynamics, become successful in the interpretation of observational data and in predicting TCS properties: multiscale structure, embedding and metastability. It become possible to solve the long-standing theoretical paradigm of magnetotail stability during substorms and explain TCS metastability, i.e. its ability to remain in a stable state for a long time, and then spontaneously change magnetic topology and initiate fast plasma acceleration and heating Thus, it is shown that TCSs are universal structures in the collisionless space plasma and they are necessary mediators of energy exchange between SW and planetary magnetospheres.

\section{Acknowledgements}

Authors are grateful to A. Artemyev for useful discussions. Work of L. Zelenyi and E. Grigorenko was supported by RSF (grant \# 16-42-01103). The work of HM was made in a frame of RFBR grant 17-02-01328, program PRAN I.24II and Volkswagen grant AZ90 312/contract 1344. The work of VP was supported by the program 28 PRAN, Program "PLASMA" and RFBR grant 16-52-16009 NCNIL.

\section{References}

[1] Biermann L and Schluter A 1951 Phys. Rev. 82863

[2] Parker E N 1958 Phys. Fluids 1171

[3] Gold T 1959 J. Geophys. Res. 641665

[4] Gringauz K, Bezrokikh V, Ozerov V and Rybchinskii R 1960 Soviet Physics Doklady 5361

[5] Ness N 1965 J. Geophys. Res. 702989

[6] Alexeev I, Belenkaya E, Kalegaev V, Feldstein Y, Grafe A 1996 J. Geophys. Res. 1017737

[7] Sergeev V, Mitchell D, Russell C and Williams D 1993 J. Geophys. Res. 9817345.

[8] McPherron R, Nishida A, Russell C 1987 Quantitative Modeling of Magnetosphere-Ionosphere Coupling Processes (Kyoto, Japan) 252

[9] Baker D Pulkinen T, Angelopoulos V, Baumjohann W and McPherron R 1996 J.Geophys. Res. 10112975
[10] Runov A, Sergeev V, Nakamura R, Baumjohann W, Apatenkov S, Asano Y, Takada T, Volwerk M, Voros Z, Zhang T, Sauvaud J-A, Reme H and Balogh A 2005 Ann. Geo. 231.

[11] Zelenyi L, Malova Kh, Artemyev A, Popov V and Petrukovich A 2011 Plas. Phys. Rep. 37118.

[12] Lui A, Lopez R, Anderson B, Takahashi K, Zanetti L, McEntire R, Potemra T, Klumpar D, Greene E and Strangeway R 1992 J. Geophys. Res. 971461

[13] Zelenyi L, Artemyev A, Malova Kh, Petrukovich A and Nakamura R 2010 Physics-Uspekhi 53933

[14] Malova H, Popov V, Grigorenko E, Petrukovich A, Delcourt D, Sharma A, Khabarova O and Zelenyi L 2017 Astrophys. J. 834 1;

[15] Zelenyi L, Oka M, Malova H, Fujimoto M, Delcourt D and Baumjohann W 2007 Sp. Sci. Rev. 132593.

[16] Zelenyi L, Malova H, Grigorenko E, Popov V 2016 Physics-Uspekhi 1861057

[17] Syrovatskii S 1971 Sov. J. Exp. Theor. Phys. 33933

[18] Frank A, Kyrie N, Markov V and Voronova E 2018

Plas. Phys. Rep. 44551

[19] Kivelson M, Bagenal F 2007 Encyclopedia of the Solar System 519

[20] Siscoe G 1975 Icarus 24311

[21] Voight G-H, Hill T and Dessler A 1983 Astrophys. J. 266390.

[22] Vasko I, Malova H, Artemyev A, Zelenyi L 2012 Planet. Sp. Sci. 9681

[23] Khurana K, Kivelson M, Vasyliunas V, Krupp N, Woch J, Lagg A, Mauk B and Kurth W 2004 Jupiter. The planet, satellites and magnetosphere 1 (Cambridge UK: Cambridge Univ. Press) 593

[24] Bagenal F 1991 Bull. Amer. Astron. Soc. 231152

[25] Zimbardo G 1989 J. Geophys. Res. 948707

[26] Kislov R, Malova Kh and Vas'ko I 2013 Moscow Univers. Phys. Bull. 6882

[27] Axford W, Petschek H and Siscoe G $1965 \mathrm{~J}$. Geophys. Res. 701231

[28] Panov E, Buchner J, Franz M, Korth A, Savin S, Reme H, Fornacon K-H 2008 J. Geophys. Res. 113 A01220

[29] Sibeck D, Lopez R and Roelof E 1991 J. Geophys. Res. 965489

[30] Nagata T 1963 Planet. Sp. Sci. 111395

[31] Bame S, Asbridge J, Felthauser H, Hones E and Strong I. 1967 J. Geophys. Res. 72113

[32] Siscoe G and Kaymaz Z 1999 J. Geophys. Res. 104 14639

[33] Chapman S. and Ferraro V 1931 Terrest. Magn. Atmos. Elec. 36171

[34] Ogilvie K, Scudder J, Vasyliunas V, Hartle R and Siscoe G 1977 J. Geophys. Res. 821807

[35] Bagenal F 2001 Planetary Magnetospheres. Encyclop. Astron. Astrophys. (Bristol UK: Inst. Phys. Publ.) 1

[36] Fujimoto M, Baumjohann W, Kabin K, Nakamura R, Slavin J, Terada N and Zelenyi L 2007 Sp. Sci. Rev. 132529 [37] Anderson B, Acuna M, Korth H, Slavin J, Uno H, Johnson C, Purucker M, Solomon S, Raines J, Zurbuchen T, Gloeckler G and McNutt R 2010 Sp. Sci. Rev 152307 
[38] Zelenyi L and Vaisberg O 1982 Cosm. Res. 20604 [39] Luhmann J, Elphic R, Russell C, Slavin J and Mihalov J 1981 Geophys. Res. Lett. 8517

[40] Vennerstrom S 2011 Icarus 215234

[41] Dubinin E, Fraenz M, Woch J, Barabash S and Lundin R. 2009 Geophys. Res. Lett. 36 L08108

[42] Vaisberg O and Zeleny L 1984 Icarus 58412

[43] Volwerk M, Delva M, Futaana Y, Retino A, Voros Z, Zhang T, Baumjohann W and Barabash S 2009 Ann. Geo. 27 2321-2330

[44] Russell C, Kivelson M, Khurana K and Huddleston D 2000 Adv. Sp. Res. 261671

[45] Vasyliunas V 1975 The magnetospheres of the Earth and Jupiter (Proc. Neil Brice Memor. Symp.: Frascati, Italy, May 28-June 1 1974) 179

[46] Grigorenko E, Shuvalov S, Malova H, Dubinin E, Popov V, Zelenyi L 2017 J. Geophys. Res. 1221

[47] Vasko I, Zelenyi L, Artemyev A, Petrukovich A, Malova H, Zhang T, Fedorov A, Popov V, Barabash S and Nakamura R. 2014 Planet. Sp. Sci. 9681

[48] Whang Y 1977 J. Geophys. Res. 821024

[49] Zelenyi L, Malova H, Popov V, Delcourt D and Sharma A 2004 Nonlin. Proc. Geophys. 11579

[50] Malova H, Popov V, Grigorenko E, Petrukovich A, Delcourt D, Sharma A, Khabarova O, Zelenyi L 2017 Astrophys. J. 8341

[51] Runov A, Sergeev V, Nakamura R, Baumjohann W, Apatenkov S, Asano Y, Takada T, Volwerk M, Voros Z, Zhang T, Sauvaud J-A, Rème H and Balogh A. 2006 Ann. Geo. 24247

[52] Asano Y, Nakamura R, Baumjohann W, Runov A, Voros Z, Volwerk M, Zhang T, Balogh A, Klecker B and Reme H. 2005 Geophys. Res. Lett. 32 L03108

[53] Malova H, Mingalev O, Grigorenko E, Mingalev I, Melnik M, Popov V, Delcourt D, Petrukovich A, Shen C, Rong Z and Zelenyi L 2015 J. Geophys. Res. 1201.

[54] Zelenyi L, Malova H, Artemyev A, Popov V, Petrukovich A, Delcourt D and Bykov A 2009 Climate and Weather of the Sun-Earth System (CAWSES, TERRAPUB: Tokyo) 121

[55] Coppi B, Laval G and Pellat R 1966 Phys. Rev. Lett. 161207

[56] Harris E 1962 Nuovo Chimento 23115

[57] Pellat R, Coroniti F and Pritchett P 1991 Geophys. Res. Lett. 18143

[58] Galeev A and Zelenyi L 1976 Transl. Zhurn. Eksperim. Teor. Fiz. 702133

[59] Karimabadi H, Daughton W, Pritchett P and KraussVarban D 2003 J. Geophys. Res. 1081400

[60] Buchner J and Kuska J-P 1999 Ann. Geophys 17604

[61] Voronkov I, Rankin R, Frycz P, Tikhonchuk V and Samson J 1997 J. Geophys. Res. 1029639

[62] Speiser T 1965 J. Geophys. Res. 704219

[63] Zelenyi L, Artemyev A, Malova H and Popov V 2008 J. Atmos. Solar Terr. Phys. 70325

[64] Zelenyi L, Artemyev A, Petrukovich A, Nakamura R, Malova H and Popov V 2009 Ann. Geo. 27861
[65] Birn J, Sommer R and Schindler K 1975 Astrophys. Space Sci. 35389

[66] Cheng C 1992 J. Geophys. Res. 971497

[67] Buchner J and Zelenyi L 1989 J. Geophys. Res. 94 11821

[68] Eastwood J 1972 Planet Space Sci. 201555

[69] Kropotkin A, Domrin V 1996 J. Geophys. Res. 101 19893

[70] Zelenyi L, Malova H, Popov V, Delcourt D and Sharma A 2004 Nonlin. Proc. Geophys. 11579

[71] Ashour-Abdalla M, Berchem J, Buchner J and Zelenyi L 1991 Geophys. Res. Lett. 181603

[72] Ashour-Abdalla M, Zelenyi L, Peroomian V and Richard K 1994 J. Geophys. Res. 9914891

[73] Artemyev A, Petrukovich A, Nakamura R and Zelenyi L 2010 J. Geophys. Res. 115 A12255

[74] Mingalev O, Mingalev I, Malova $\mathrm{Kh}$ and Zelenyi L 2007 Plas. Phys. Rep. 33942

[75] Delcourt D, Malova H and Zelenyi L 2004 J.Geophys.Res. 109 A01222

[76] Zelenyi L, Delcourt D, Malova H and Sharma A 2002 Geophys. Res. Lett. 29 49-1

[77] Sitnov M, Zelenyi L, Malova H and Sharma A $2000 \mathrm{~J}$. Geophys. Res. 10513029

[78] Zelenyi L, Sitnov M, Malova H and Sharma A. 2000 Nonlin. Proc Geophys. 7127

[79] Zelenyi L, Malova H, Popov V, Delcourt V, Ganushkina N and Sharma A 2006 Geophys. Res. Lett. 33 L05105

[80] Nakamura R, Baumjohann W, Runov A and Asano Y 2006 Sp. Sci. Rev. 12229

[81] Malova H, Zelenyi L, Popov V, Delcourt D, Petrukovich A and Runov A. 2007 Geophys. Res. Lett. 34 L16108

[82] Schindler K 1979 Space Sci. Rev. 23365

[83] Lembege B, Pellat R 1982 Phys. Fl. 251995

[84] Pritchett P 2005 J. Geophys. Res. 110 A10213

[85] Daughton W 2002 Phys. Plas. 93668

[86] Sitnov M, Swisdak M, Drake J, Guzdar P and Rogers B 2004 Geophys. Res. Lett. 31 L09805

[87] Birn J, Hesse M and Schindler K 2006 Sp. Sci. Rev. 124103

[88] Zelenyi L, Kropotkin A, Domrin V, Artemyev A, Malova H and Popov V 2009 Cos. Res. 47352 